\title{
CLASSICAL AND QUANTUM CELL-CELL SIGNALING BY MICROBIAL LIFE ON EARTH AND POSSIBLE OTHER LIVABLE WORLDS
}

\author{
Kevin B. Clark, Ph.D. \\ (kbclarkphd@yahoo.com and kevin.clark@mpg-alumni.de)
}

Director, Felidae Conservation Fund, Mill Valley, CA 94941, USA; Co-Chairperson, Science Advisory Board, Cures Within Reach, Chicago, IL 60602, USA; Domain Champion in Biomedicine and Campus Champion, NSF Extreme Science and Engineering Discovery Environment (XSEDE), National Center for Supercomputing Applications, University of Illinois at Urbana-Champaign, Urbana, IL 61801, USA; Affiliate, NASA Ames Research Center, Mountain View, CA 94035, USA; Affiliate, NASA NfoLD, NASA Astrobiology Program, NASA Ames Research Center, Mountain View, CA 94035, USA; Advisor, Universities Space Research Association, Columbia, MD 21046, USA; Subject Matter Expert and Mentor, Penn Center for Innovation, University of Pennsylvania, Philadelphia, PA 19104, USA; Consultant and Collaborator, Research and Development Service, Veterans Affairs Greater Los Angeles Healthcare System, Los Angeles, CA 90073, USA; Main Organizer, Shared Interest Group for Natural and Artificial Intelligence (sigNAI), Max Planck Alumni Association, 14057 Berlin, Germany; Member, Nanotechnology and Biometrics Councils, Institute for Electrical and Electronics Engineers (IEEE), New York, NY 10016-5997, USA.

White Paper Running Head: ClarkKevinB3, Cell-Cell Signaling by Earth and Extraterrestrial Microbes 


\begin{abstract}
Cell-cell signaling, a fundamental innovation of early social life forms, helps Earth microbes adapt to varying ambient and host eco-evolutionary settings. Improved scientific study and understanding of signaling traits will reveal aspects of possible extraterrestrial microbial social behaviors impacting other extinct or extant livable worlds.
\end{abstract}

\title{
BACKGROUND
}

Overcoming errors in communications and information processing is an endemic problem for all biological systems. For "social" microbes capable of quite advanced cell-cell signaling and cellular decision making, the necessity of error-syndrome diagnosis and correction can be just as important to survive and reproduce in hostile environments as it is for phylogenetically more recent social animals and "higher" plants (1-22). Fidelity of processed information may predictably determine, for instance, whether colonial prokaryotes and "lower" eukaryotes successfully communicate deceptive or honest signals to conspecifics while instigating and/or mediating conflicts that secure opportunities for improved individual or group ecological fitness, such as group nutrient foraging or hunting, paired reproduction, and collective defenses against predation and environmental stressors/hazards. Indeed, (natural and/or directed) selected aptitudes and aparati for communications enable microbes to further innovate and evolve social attributes with the potential capacity to shape the eco-evolutionary trajectories of niches to biospheres. Despite the great value for astrobiology to better understand the origins, nature, and evolution of cellular life from solitary single cells to complex social structures and processes, little to no attention has been given to how microbes communicate within and across tava and how they secure their cell-cell communications from corruption by ambient Earth and/or possible extraterrestrial environmental noise and from eavesdropping by unintended observers.

Microbial cell-cell signaling may occur within a wide variety of social or social-like contests. Obvious useful examples of model social contexts include nonclonal reproductive situations, which have profound implications for eco-evolutionary transitions on Earth and possibly elsewhere in the universe. The mating mechanisms of microbes across systematics differ considerably, but general commonalities in chemical signals, ostensive and nonostensive behavioral "rituals", and reproductive goals support the hypothesis that mate selection, itself a kind of fault inspection employed to lower the probability of genetic exchange with defective partners, originated before the evolutionary divergence of animals and fungi $(3-10,12,14)$. As with animals, microbial strategies of intra- and intermate selection advance cohort and offspring adaptation through nonpromiscuous unions that minimize survival-reproductive tradeoffs. Making mate choices based on the perceived ecological fitness of suitors advertising superior somatic structures (e.g., ornaments and weapons), motility competence (e.g., courtship dances), social aptitude (e.g., conflict mediation and instigation), and/or other survival characteristics also promotes the vertical and, where applicable, horizontal spread of beneficial inherited traits, such as tolerance to stressors and toxins, pathogen virulence, biofilm formation, and cell aggregation, within subdivided populations at greater rates and efficiencies. Although microbial mate selection appreciably favors virulence and transmission of infectious diseases, major evolutionary transitions, and emergence of primitive social intelligences, the biological and 
computational processes used by microbes to identify and correct performance faults during mate selection remain, with few exceptions, poorly understood $(3-10,12,14)$.

\section{COMMUNICATION TRAITS OF MODEL EARTH SOCIAL MICROBES - CILIATED PROTOZOA}

Intra- and intermate selection can be complex sequences of events in prokaryotes and microbial eukaryotes, with each stage susceptible to error in intracellular information processing and/or cell-cell communication (3-10,12,14). Many ciliates, for example, detect and respond to peptide pheromones secreted by nonself mating types. Pheromones announce the location and type of mate(s) available. Sufficiently attracted individuals may try to engage one or several compatible partners (i.e., an opposite mating type) in sexual-like conjugation normally producing eight daughter cells per reproductive pair at lifecycle's end. In different ciliate genera, stereotypic courtship dances, dominance displays, and preconjugal changes in cell structure allowing partner docking and gene exchange result from specific pheromone binding to extracellular domains of G-protein-associated paracrine receptors. And like other eukaryotic microbes, activation of sexual pheromone transduction pathways in ciliates is further modified by cell-cell contacts and related events that initiate intracellular $\mathrm{Ca}^{2+}$-induced $\mathrm{Ca}^{2+}$ cascades and phosphatase- and proteinkinase-dependent feedback regulation of mechanosensation and learned motility. The oftentimes "programmed" or instinctual-like character of ciliate matings would suggest that fault-tolerance is built into the structure and function of relatively inflexible genetic and epigenetic regulatory networks controlling signal coding, planning, execution, and decryption during preconjugal activities. But experiments simulating mating contexts have elaborated traditional learned aspects of ciliate intra- and intermate selection, indicating nonassociative and associative learning and memory processes may substantially contribute to detecting and correcting information errors (3-10,12.14). To facilitate mating success, heterotrich contractile ciliates, for example, learn to advertise various degrees of mating fitness to perceived "suitors" and "rivals" by serially contracting or ciliary reversing at rates that signal either conspicuous consumption or prudent savings.

Ciliates exhibiting responsiveness of conspicuous consumption declare fit reproductive status via the excessive rates at which they signal avoidance. Superior mating candidates are alone capable of metabolically wasteful displays. Since the behavior of conspicuous consumers makes it difficult for exchange of preconjugal touches between courting couples, they play "harder-toget" when responding to the presumed advances of nearby conspecifics. Prudent savers, on the other hand, conserve energy stores for scenarios more favorable for conjugating a partner. These ciliates reply with lower frequencies of avoidance reactions, guaranteeing would-be fellow ciliates of the probability of being "easier-to-get” during courtship dances. By deciding to switch from an initial behavioral strategy that signals conspicuous consumption to one that signals prudent savings, fitter ciliates learn to altruistically sacrifice potential net payoffs to persuade suitors to participate in paired reproduction. In effect, they incur higher reproductive costs, in terms of possible offspring numbers and viability, by mating with inferior ciliates. Less fit ciliates unable to sustain long periods of high response rates may switch their initial behavioral strategy of prudent savings to briefly emit conspicuous consumption and thus learn to increase net payoffs by opportunistically cheating finer rivals and engaging higher quality suitors in conjugal activity. 


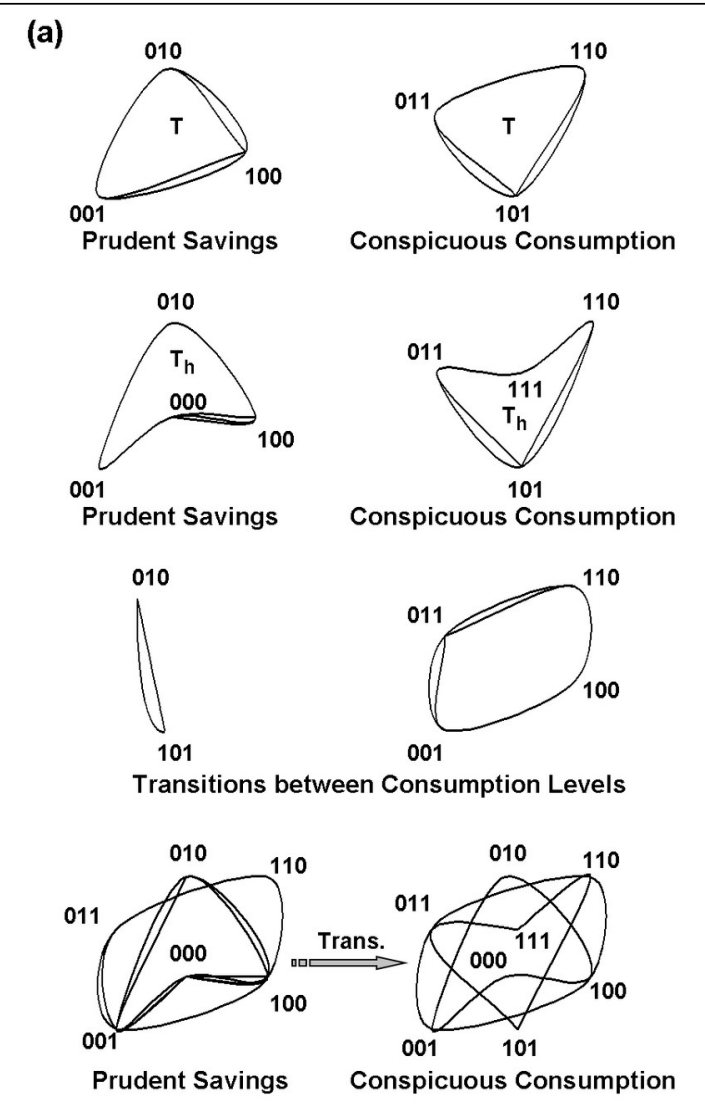

(b)

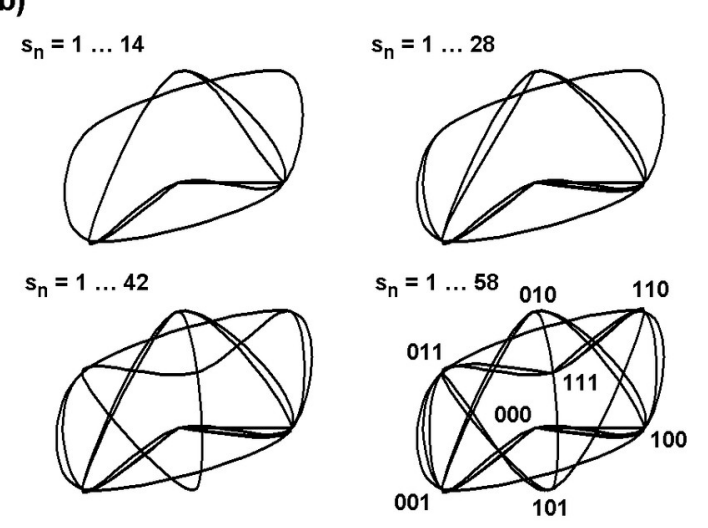

(c)
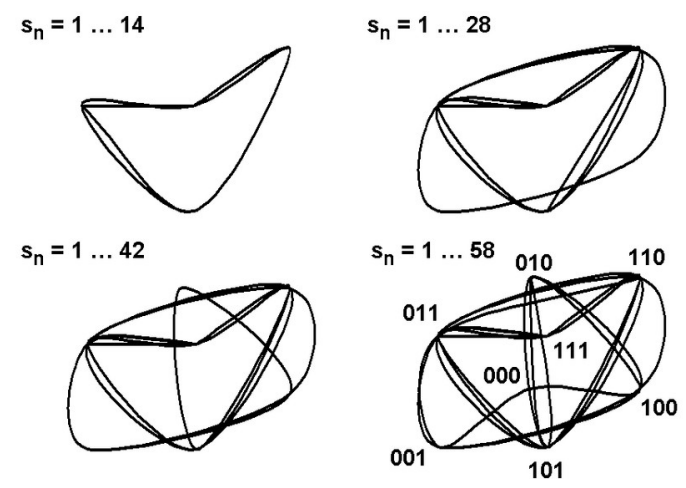

Figure 1. Computational structure of signaling heuristics. Ciliates build heuristics from grouped serial behavioral strategies used to signal courting assurances. (a) Serial contraction or reversal strategies containing three consecutive behavioral events, symbolized by binary strings composed of basis bit 1 (response) and/or 0 (no response), organize into several types of homology groups. Serial strategies with one or fewer responses convey prudent savings. Those with two or more responses convey conspicuous consumption. Topologically invariant groups of tetrahedral-like ( $\mathrm{T}$ ) (top row) and pyritohedral-like $\left(\mathrm{T}_{\mathrm{h}}\right)$ (second row) symmetry store different signals of uniform mating fitness. Groups storing different signals of mixed mating fitness make transitions between strategies of prudent savings and conspicuous consumption (third row). An incomplete heuristic used by a ciliate transitioning from prudent savings to conspicuous consumption during sensitization learning (bottom row). (b), (c) Examples of multistable contraction and ciliary reversal heuristics evolving over time, $s_{n}$, into 3D computational networks with eight different strategies. The same ciliate built, searched, and used both heuristics. It began testing by signaling prudent contraction savings and conspicuous reversal consumption, then sensitized its contraction output to signal conspicuous consumption and habituated its reversal output to signal prudent savings. Edges between vertices of computational structures in panels (a) through (c) indicate a ciliate's choice to switch from one signal to another. Recurrent decisions overlap and might not be visible in heuristic representations. Figure 1 reproduced from (4) with permission.

The ability of each heterotrich ciliate to appropriately stay with the same reply or to switch its reply from one behavioral strategy to another critically depends on the type of dual-process 
nonassociative learning expressed (i.e., sensitization or habituation), the duration of learning (i.e., longer- or shorter-term), and the efficiency of heuristics formed from recursive strategy searches and use. Heuristics represent stored patterns of action used by a ciliate. They evolve via classical Maxwell-Boltzmann (MB), quantum Bose-Einstein (BE), and quantum Fermi-Dirac (FD) statistics into ordered computational networks of serial escape behaviors organized around centers of smaller, local strategy groups supporting courting assurances of harder-to-get and easier-to-get (3-10,12-15). Computation networks exhibit some degree of fault tolerance through topological invariance (see Figure 1). Furthermore, as ciliates develop their signaling skills over numerous trials, the connectivity between different strategies often strengthens from Hebbianlike learning which, in turn, promotes faster and less error-prone decisions about the quality of mating replies until a single solution is found (see Figure 2). The time taken by the best experts to master signaling decisions achieves efficiencies that resemble the quantum process of finding target solutions with Grover's search algorithm. Very successful strategy searches coincide with emergence of computational analogues of Bose-Einstein condensation in signal choice and of quantum tunneling in decision rates. These quantum computational phenomena are consistent with organic chemical reactions thought to produce weak Fröhlich condensation and quantum tunneling at physiological temperatures as well as with the operation of classical and quantum mechanochemical engines that obey heat and refrigeration statements derived from the laws of thermodynamics $(3-6,13,14)$.
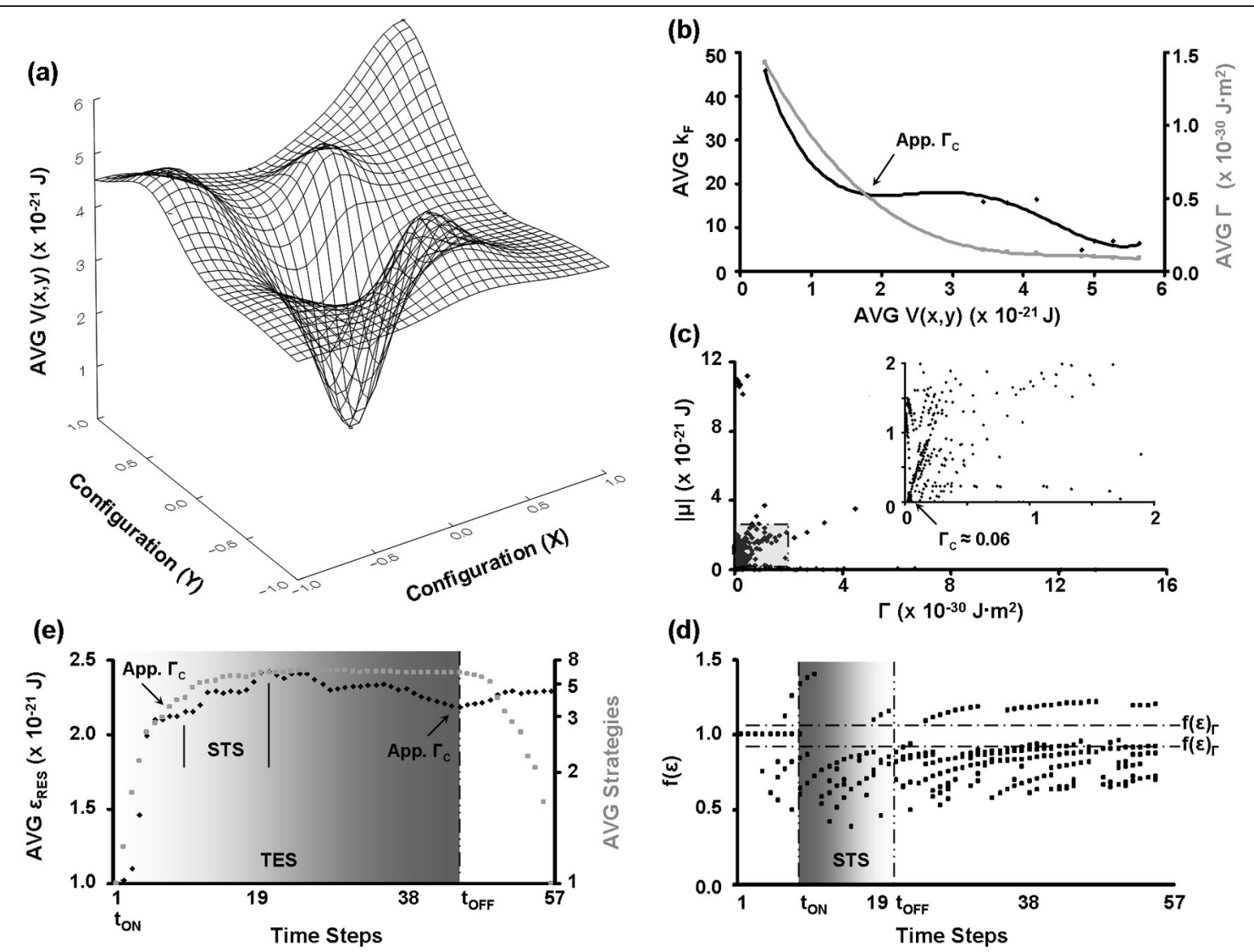

Figure 1. Prudent savers beginning condensed on single 'courting' solutions sensitized contraction rates to signal Maxwell-Boltzmann phase of 'harder-to-get'. (a) Potential landscape averaged across all initial prudent savers $(n=14)$ displaying shorter-term sensitization 
(STS) of contraction responses. Potential $V(x, y)$ equaled energy level $\varepsilon$ for each heuristic node corresponding to a serial contraction strategy. 3D structure of each heuristic was transformed into an isomorphic xy configuration for graphical depiction of landscape. Landscape reached deepest well at reconfigured coordinate $(0,0)$ mapping onto modal strategy 000 signaling prudent 'easier-to-get'. (b) Average final connectivity $\left(k_{\mathrm{F}}\right)$ for heuristic nodes produced a fourthorder polynomial distribution (black line). Most connections (i.e., decisions) occupied the lowest average potential well of modal strategy 000. BE condensation for new links appeared below the approximate 'critical tunneling field strength', App. $\Gamma_{\mathrm{C}}$. Average initial $\Gamma$ (grey line) was proportional to $\varepsilon$, but decreased as energy levels filled with connections. Heuristic nodes gathering about twenty links transitioned from computational phases vulnerable to thermodynamic limits into a BE condensate. (c) A precise plotting of $|\mu|$ against $\Gamma$ determined $\Gamma_{\mathrm{C}}$ for modal strategy 000 to be an order of magnitude lower than App. $\Gamma_{\mathrm{C}}$ in (b). Inset shows exploded view of area within grey-shaded box. (d) Rate, $f(\varepsilon)$, at which the heuristic node corresponding to modal strategy 000 acquired new links demonstrated ciliates began testing condensed on a single 'courting' solution falling within the critical interval, $f(\varepsilon)_{\Gamma}$. Decisions to use this strategy changed with onset of STS. STS reset the BE computational phase to one largely governed by MB statistics and other nodes of higher energy levels corresponding to conspicuous 'harder-to-get' signals. Offset of STS accompanied a return to $f(\varepsilon)$ characteristic of $\mathrm{BE}$ statistics and favored use of modal strategy 000. (e) Average residual energy, $\varepsilon_{\mathrm{RES}}$ (black dotted line), indicated annealing coincided with recursive trial-and-error strategy searches (TES). Ciliates began testing at lowest possible AVG $\varepsilon_{\text {RES }}$ consistent with being condensed on a single 'courting' solution. STS reset average $\varepsilon_{\text {RES }}$ to higher levels as average number of strategies used by ciliates increased (grey dotted line). With offset of STS, TES annealed $\varepsilon_{\text {RES }}$ to a minimum near App. $\Gamma_{\mathrm{C}}$, where BE condensation reduced average number of strategies employed. Figure 2 reproduced from (4) with permission.

Notably, the engine-like traits of intracellular bioprocesses mediating ciliate social decision making offer suitable means to limit faults and improve individual or shared ecological advantages through identification and recovery of information corrupted during noisy stages of signaling performance and message transmission, much like comparator routines implemented by artificial and hybrid technological systems (3-8,10,12,14-16). To implement error diagnosis and correction procedures, information coding schemes become necessary. Clark (3-8,10,12-15) first proposed unique anticorrelated bit-string configurations of modal behavioral signals emitted by ciliates enables construction classical repetition and possibly superposed quantum bit-flip error-correction codes that maintain specificity of cell-cell communications as well as help prevent deterioration of mating signals from both color and white environmental noise. These sorts of classical and quantum codes contain redundant bit and qubit states which lower the probability that socially important transmitted information could be irretrievably lost to decoding. Such codes expect to be valuable in selectively conveying net reproductive fitness to upwards of hundreds of different viable mating types located in geographically constricted areas, where, for instance, isolation from conspecifics is low and likelihood of acquiring deleterious genes is high.

\section{CONCLUSIONS}

Many of the survival and reproductive achievements of colonial bacteria, algae, fungi, and 
protozoa, like other kinds of organisms, are dependent upon genetically predisposed, epigenetically modifiable, and traditionally learned social skills that enhance an individual microbe's ability to communicate and interact with others. Good social skills often make groups of microbes more adaptable through coordinated hunting and foraging, mate selection, altruistic suicide, assisted reproduction, induced defenses, and additional behaviors (1-22). But without proper methods to control errors in social interactions, the ecological success of social species, subspecies, collectives, kin, and individuals would deteriorate to levels indistinct from those of solitary organisms and, importantly, would likely serve as a barrier for transitions to multicellualrity and other eco-evolutionary significant events. Although computational faulttolerance figures to substantially impact a number of microbial behaviors, such as intra- and intermate selection, which contribute to major eco-evolutionary transitions, astrobiologists have made little progress in elucidating these crucial aspects of fast, efficient cell-cell signaling and information processing in early life forms. Ciliates and perhaps other microbial taxa learn to diagnose and correct classical and possibly quantum information errors incurred throughout the production of serial behavioral strategies useful for signaling mating availability and prowess during perimating displays (3-14). Moreover, similar error-syndrome diagnosis and correction procedures likely safeguard the information content and specificity of other forms of ciliate behavioral communications and might be universally used by all microbes during social situations. Mechanosensation, while mediated by disparate transduction mechanisms, is a phylogenetically conserved sensory modality of single-celled organisms. Bacteria, algae, molds and fungi, and protozoa other than heterotrich ciliates also rely on mechanosensation for sensing osmotic stress, prey or predators, reproductive interactions, foraging, cell aggregation, surface contours, and a variety of additional cell physiologic and ecological conditions. Chemical communications, which might be used by astrobiologists as biosignatures for extraterrestrial life detection, serve as a primary means for microbes to exchange social information (e.g., 2,912,15,19-22), but high-fidelity mechanical communication is nonetheless critical for coordinating collective behaviors and the spatial organization of homo-/heterogeneous microbial groups. Use of classical and quantum codes by microbes to protect serial mechanical messages and replies, alone or in combination with chemicals and/or additional communication signals (e.g., electromagnetic signals), from ambient noise and social eavesdroppers represents a possible widespread eco-evolutionary adaptation in the computational abilities of bacteria to protozoa and perhaps life elsewhere in the universe. Future work should better evaluate such phenomena in relation to the social, physiologic, and environmental conditions necessary to help originate and shape Earth and extraterrestrial planetary niches to biospheres.

\section{REFERENCES}

1) Ben-Jacob, E. (2008). Social behavior of bacteria: From physics to complex organization. European Physics Journal B, 65, 315-322.

2) Ben-Jacob, E., Becker, I., Shapira, Y., Levine, H. (2004). Bacterial linguistic communication and social intelligence. Trends in Microbiology, 12, 366-372.

3) Clark, K.B. (2010). Origins of learned reciprocity in solitary ciliates searching grouped 'courting' assurances at quantum efficiencies. BioSystems, 99(1), 27-41.

4) Clark, K.B. (2010). Bose-Einstein condensates form in heuristics learned by ciliates deciding to signal 'social' commitments. BioSystems, 99(3), 167-178.

5) Clark, K.B. (2010). On classical and quantum error-correction in ciliate mate selection. 
Communicative \& Integrative Biology, 3(4), 374-378.

6) Clark, K.B. (2010). Arrhenius-kinetics evidence for quantum tunneling in microbial "social" decision rates. Communicative \& Integrative Biology, 3(6), 540-544.

7) Clark, K.B. (2012). Social biases determine spatiotemporal sparseness of ciliate mating heuristics. Communicative \& Integrative Biology, 5(1), 3-11.

8) Clark, K.B. (2013). Ciliates learn to diagnose and correct classical error syndromes in mating strategies. Frontiers in Microbiology, 4, 229.

9) Clark, K.B. (2013). Biotic activity of $\mathrm{Ca}^{2+}$-modulating nontraditional antimicrobial and -viral agents. Frontiers in Microbiology, 4, 381.

10) Clark, K.B. (2013). The mating judgments of microbes. In K.B. Clark (Ed.), Social learning theory: Phylogenetic considerations across animal, plant, and microbial taxa, pp. 173-200. Hauppauge: Nova Science Publishers, Inc. ISBN 978-1-62618-268-4.

11) Clark, K.B. and Eisenstein, E.M. (2013). Targeting host store-operated $\mathrm{Ca}^{2+}$ release to attenuate viral infections. Current Topics in Medicinal Chemistry, 13(16), 1916-1932.

12) Clark, K.B. (2014). Evolution of affective and linguistic disambiguation under social eavesdropping pressures. Behavioral and Brain Sciences, 37(6), 551-552.

13) Clark, K.B. (2014). Basis for a neuronal version of Grover's quantum algorithm. Frontiers in Molecular Neuroscience, 7, 29.

14) Clark, K.B. (2015). Insight and analysis problem solving in microbes to machines. Progress in Biophysics and Molecular Biology, 119, 183-193.

15) Clark, K.B. (2018). Searching for (Proto)Cellular Logics Proteins in Earth-like Environments Hospitable and Inhospitable to Life. White paper submitted to the Committee on an Astrobiology Science Strategy for the Search for Life in the Universe, National Research Council, Washington, D.C.

16) Clark, K.B. (2019). Unpredictable homeodynamic and ambient constraints on irrational decision making of aneural and neural foragers. Behavioral and Brain Sciences, 42, e40.

17) Clark, K.B. (2019). Neurotropic enteroviruses coopt "fair-weather-friend" commensal gut microbiota to drive host infection and CNS disturbances. Behavioral and Brain Sciences, 42, e68.

18) Crespi, B.J. (2001). The evolution of social behavior in microorganisms. Trends in Ecology and Evolution, 16, 178-183.

19) Koseska, A., Zaikin, A., Kurths, J., Garciá-Ojalvo, J. (2009). Timing cellular decision making under noise via cell-cell communication. PLOS ONE, 4, e4872.

20) Ricci, N. (1990). The behavior of ciliated protozoa. Animal Behavior, 40, 1048-1069.

21) Trewavas, A. (2003). Aspects of plant intelligence. Annals of Botany, 92, 1-20.

22) Winans, S.C., Bassler, B.L. (2002). Mobpsychology. Journal of Bacteriology, 184, 873-883. 